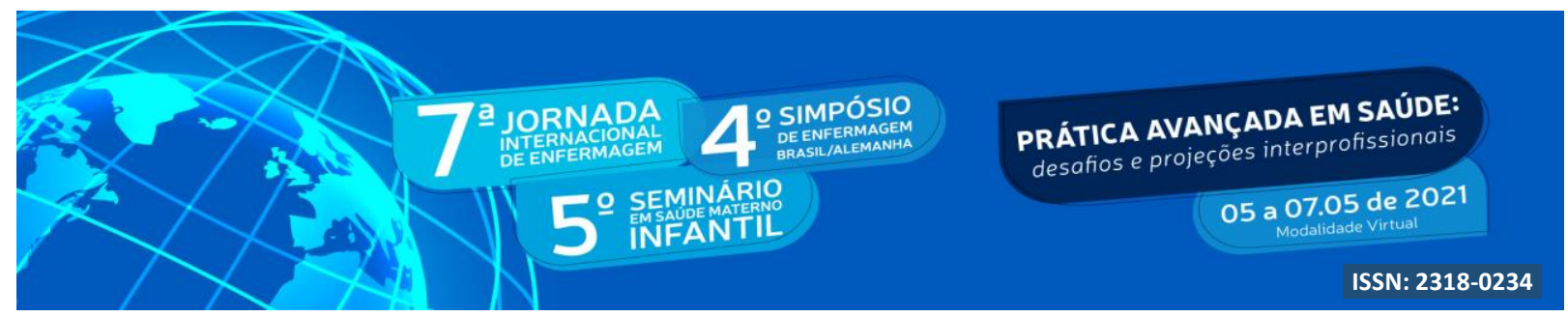

DOI: http://doi.org/10.48195/jie2021-085

\title{
IMPORTÂNCIA DAS BOLSAS DE INICIAÇÃO CIENTÍFICA PARA A FORMAÇÃO ACADÊMICA ${ }^{1}$
}

\author{
André Vernier Stochero ${ }^{2}$; Andreza Cossettin de Souza ${ }^{3}$; Karol Trevisan Sartori ${ }^{4}$; \\ Jucimara Montagner Michelon ${ }^{5}$; Cláudia Zamberlan ${ }^{6}$
}

\begin{abstract}
RESUMO
A iniciação científica torna-se importante para estimular os acadêmicos desde o início do curso, no intuito de fazê-los participar, aprimorar e dedicar-se às pesquisas sobre diferentes temáticas e ênfases. Objetiva-se relatar a experiência discente com a iniciação científica para formação acadêmica. Configura-se como uma reflexão teórica, baseada na literatura científica sobre a importância da iniciação científica na trajetória acadêmica construída no primeiro bimestre do ano de 2021, com o suporte teórico das evidências científicas publicadas nos últimos 5 anos e elaborado por acadêmicos do curso de graduação em Enfermagem, bolsistas de Iniciação Científica de uma Universidade privada localizada na região central do Rio Grande do Sul. Conclui-se que a iniciação científica é um instrumento relevante para a formação, pois é por meio dela que os estudantes de graduação se inserem na pesquisa científica resultando na aplicação dos conhecimentos para a aplicabilidade prática.
\end{abstract}

Palavras-chave: Bolsa de estudos; Ciência; Educação Superior; Enfermagem.

\begin{abstract}
The scope of scientific initiation is important because it stimulates undergraduates since their first years attending their major, enable them to participate, improve and dedicate themselves to research in different topics. The current research consists in a theoretical reflection based on the scientific literature towards evidences on the importance of scientific initiation during the academic trajectory, developed by academics of the fifth, nineth and tenth semesters of the Nursing major who are scientific initiation scholars of PROBIC - Fapergs, PIBIC -CNPq and PROBIC - UFN from the

\footnotetext{
${ }^{1}$ Reflexão teórica.

${ }^{2}$ Acadêmico do curso de Enfermagem - Bolsista PROBIC-UFN. Universidade Franciscana. E-mail: andrevstochero@gmail.com

3 Acadêmica do curso de Enfermagem - Bolsista PROBIC-Fapergs. Universidade Franciscana. E-mail: andrezacossettin@gmail.com

${ }^{4}$ Acadêmica do curso de Enfermagem - Bolsista PIBIC-CNPq- Universidade Franciscana. E-mail: karol.sartori@ufn.edu.br

${ }^{5}$ Enfermeira. Mestranda em Saúde Materno Infantil- Universidade Franciscana. E-mail: jucimara.michelon@ufn.edu.br

${ }^{6}$ Orientadora. Docente do curso de Enfermagem. Universidade Franciscana. Email: claudiaz@ufn.edu.br
} 


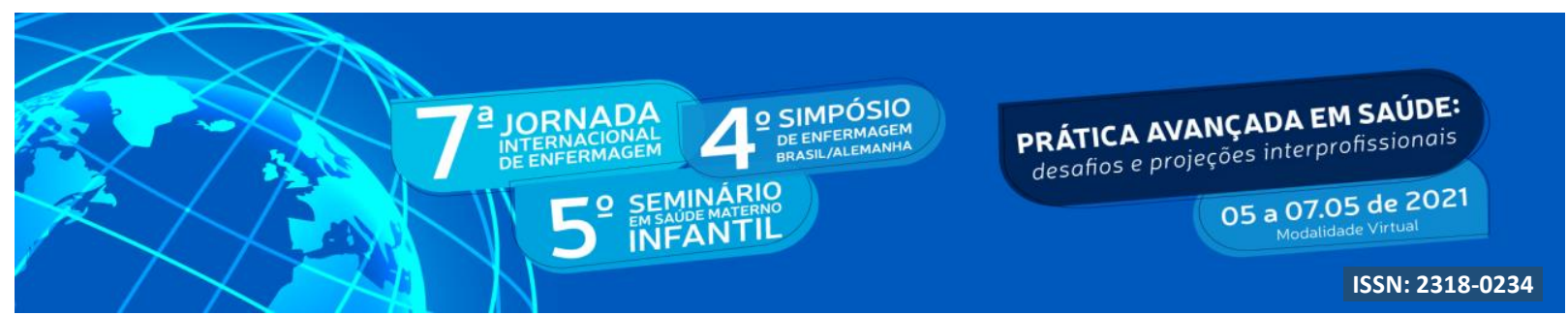

Franciscan University of Santa Maria, RS (Universidade Franciscana). This research aims at reporting undergraduates'scientific initiation experiences and its implications to their academic development. It is a theoretical reflection based on the scientific literature developed in the first two months of the year 2021, with the theoretical support of the scientific evidences published in the last five years.

Key Words: College fund; Science; Higher education; Nursing.

\section{INTRODUÇÃO}

A Enfermagem enquanto ciência e profissão, no contexto atual é uma área que tem produzido muito no âmbito científico e isso colabora com a prática vivenciada pelos estudantes da graduação os quais podem ser formadores de novos conhecimentos (QUEVEDO; GIMENEZ, 2019).

A iniciação científica (IC) torna-se importante para estimular os estudantes desde o início do curso, no intuito de fazê-los participar, aprimorar e dedicar-se às pesquisas sobre diferentes temáticas e ênfases, visto que, isso será relevante para sua formação, não somente no contexto profissional como também pessoal e social. Deste modo, os estudantes são estimulados a refletirem mais sobre diferentes temas e, a serem mais questionadores frente a novos conhecimentos, fomentando aprender mais sobre noções teóricas e metodológicas e posteriormente agregar no currículo (QUEVEDO; GIMENEZ, 2019).

Nesse constructo, os grupos de pesquisa oferecem aos estudantes a oportunidade de desenvolvimento de habilidades e o ganho de maturidade pessoal e profissional a partir da experiência em projetos de pesquisa e de extensão. Além disso, destaca-se a importância do trabalho em equipe, do estímulo e operacionalização com foco na busca de soluções para problemas encontrados na prática profissional e na troca de experiências com os estudantes e professores, assim como, o estímulo no desenvolvimento de novas pesquisas na área da saúde e enfermagem (AZEVEDO et al., 2018).

A IC é um dispositivo usado para inserir os estudantes de graduação na pesquisa científica visando a formação de recursos humanos qualificados. No decorrer do curso de enfermagem, a IC estimula o aluno a aproximar-se da realidade social, encorajando-o a desenvolver pesquisas ainda no meio acadêmico e colaborando tanto para a formação 


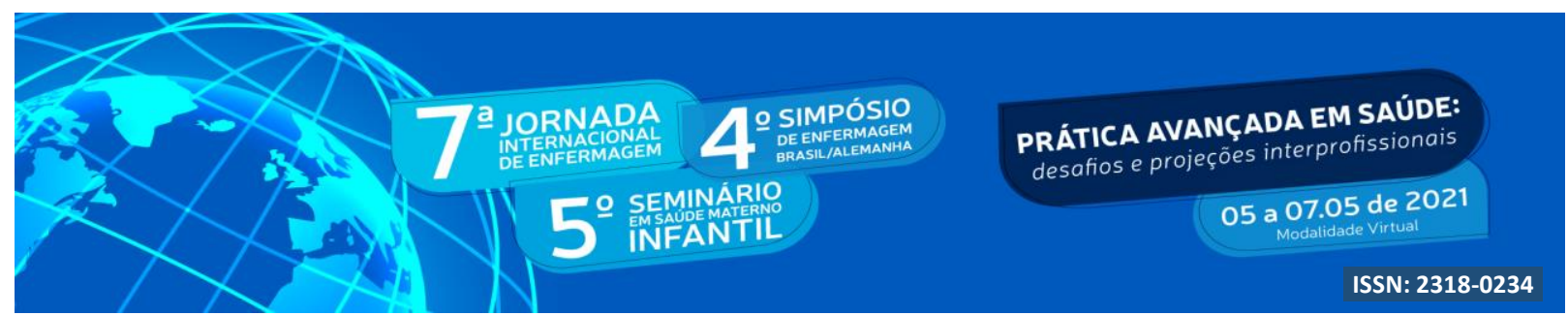

profissional dos estudantes como para a ciência e sociedade. (SANTOS; ANJOS; ALMEIDA, 2015).

Destaca-se ainda que a IC é um importante instrumento para desenvolver um princípio ético e profissional, destacando-se como modalidade de estudo na qual proporciona ao aluno da graduação despertar a vocação para a pesquisa e incentivar estudantes em potencial. É um espaço da pesquisa científica designado para estudantes de graduação para que possam elaborar projetos e aprimorar conhecimentos, estando assim mais preparados para continuar sua trajetória acadêmica (PEREIRA et al., 2015). Com base nesse enfoque e considerando a importância dos princípios da IC para a formação acadêmica destaca-se como objetivo desse trabalho:

\section{OBJETIVO}

Relatar a importância da iniciação científica para a formação acadêmica.

\section{METODOLOGIA}

Trata-se de uma reflexão teórica, baseada na literatura científica acerca das evidências sobre a importância da IC na trajetória acadêmica, elaborado por acadêmicos do curso de graduação do $5^{\circ}, 9^{\circ}$ e $10^{\circ}$ em Enfermagem, bolsistas de Iniciação Científica PIBIC- CNPq, PROBIC- UFN e PROBIC- Fapergs, respectivamente, de uma Universidade localizada na região central do Rio Grande do Sul, na cidade de Santa Maria.

Primeiramente, foi realizada a leitura de artigos relacionados com o tema, após, podese dar início a escrita dessa reflexão teórica no primeiro bimestre do ano de 2021. Foram selecionados materiais científicos publicados nos últimos 5 anos para a leitura, análise das informações e discussão dos resultados.

\section{RESULTADOS E DISCUSSÃO}




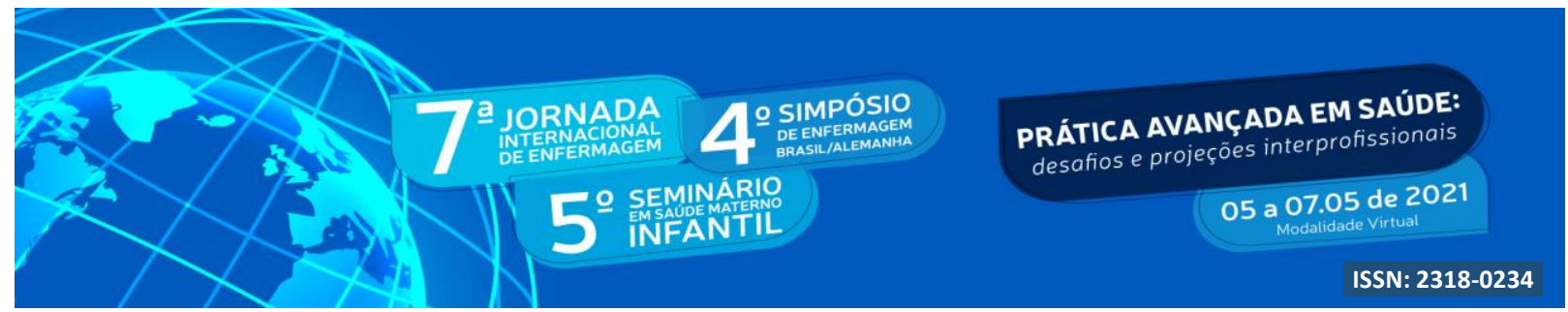

$\mathrm{Na}$ Enfermagem, a pesquisa científica objetiva o aperfeiçoamento do bem-estar de vida da população e pode ser caracterizada como um processo de produção e reprodução do conhecimento. É de grande relevância compreender o ensino da pesquisa na graduação com o propósito de formar enfermeiros qualificados e fortalecer a Enfermagem como ciência em construção, visibilizando a profissão (MORAES et al., 2018).

A introdução do aluno de graduação no mundo da ciência, das técnicas científicas e do desenvolvimento de projetos de pesquisa ocorre sob a orientação de um docente (PINHO, 2017). Destaca-se ainda que a trajetória acadêmica é consolidada como um momento importante para direcionar a atividade de pesquisa a novos níveis, proporcionando maior desenvolvimento de competências e habilidades que garantem ao enfermeiro refletir criticamente e com ciência a sua profissão futuramente (MORAES et al., 2018).

A incorporação dos estudantes em projetos de pesquisa, contribui no processo educacional, como também, no favorecimento das relações interpessoais e interdisciplinares, além de proporcionar o alcance de novos conhecimentos e aprendizado científico. Mediante este método, os graduandos podem obter conhecimentos que ultrapassam o da graduação, ponderando na vida profissional futura (SOBRAL; SANTOS; TORALES, 2016).

Por vezes, advém a carência de novos discentes motivados em participar da iniciação científica, acrescido a isso, ainda, existem pesquisas que mostram continuidade de estratégias e objetos de estudo para novos projetos, razões na qual levam os professores orientadores a renovarem os contratos com os mesmos discentes (PINTO; FERNANDES; SILVA, 2016).

$\mathrm{O}$ docente atua como orientador e incentivador desta experiência por meio das atividades que instigam e orientam o estudante. Nesse ínterim é necessário profissionais com caráter inovador, com referenciais metodológicos que busquem na essência dos discentes e a melhor metodologia para desenvolver a aprendizagem. (OLIVEIRA; SILVA; ALBUQUERQUE, 2016).

\section{CONCLUSÃO}




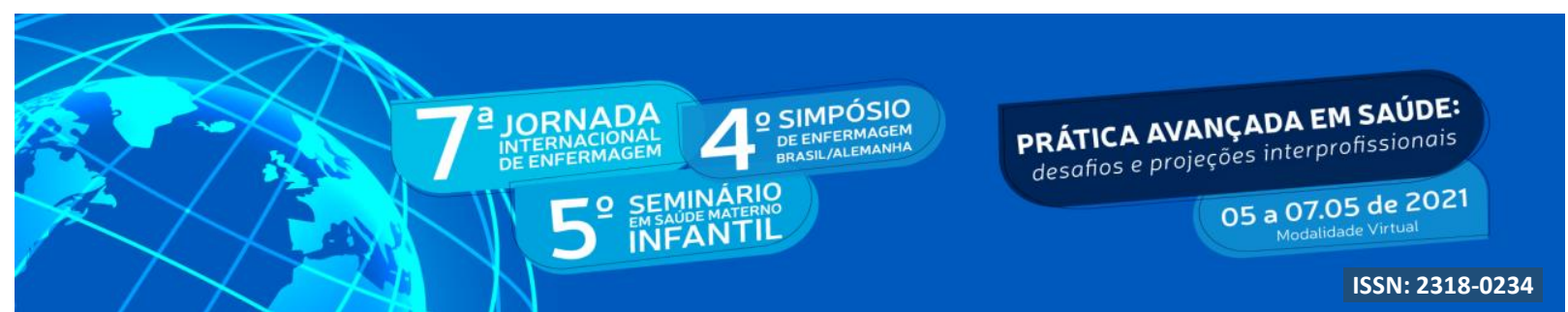

Conclui-se que a IC é um instrumento muito importante para a formação acadêmica, pois é por meio dela que os estudantes de graduação se inserem na pesquisa científica resultando na aplicação dos conhecimentos com maior clareza. Ainda se destaca a relevância de vivenciar essa oportunidade que a graduação oferece, pois o profissional que executa a prática por meio das evidências cientificas tem resultados melhores em seu processo de trabalho.

Participar da bolsa de IC dentro da graduação é uma perfeita oportunidade para que o estudante se desenvolva no meio científico. Além disso, consegue associar a teoria com a prática, e assim compreender algumas questões em que ficaram com dúvidas. Por fim, a inserção de alunos em projetos, favorece o processo educativo por parte dos docentes, beneficiando assim as duas partes.

\section{REFERÊNCIAS}

AZEVEDO, I. C.; SILVA, R. C. L.; CARVALHO, D. P. S. R. P.; CRUZ, G. K. P.; LIMA, J. V. H.; JÚNIOR, M. A. F. Importância do grupo de pesquisa na formação do estudante de enfermagem. Rev Enferm UFSM. v. 8, nº. 2, pág. 390-398, Abril/Junho de 2018. Disponível em: https://periodicos.ufsm.br/reufsm/article/view/26003/pdf. Acesso em: 03 março de 2021.

MORAES, A.; GUARIENTE, M. H. D. M.; GARANHANI, M. L.; CARVALHO, B. G. A formação do enfermeiro em pesquisa na graduação: percepções docentes. Rev Bras Enferm. v. 71, no 4, pág. 1648-1656, 2018. Disponível em:

https://www.scielo.br/pdf/reben/v71s4/pt_0034-7167-reben-71-s4-1556.pdf. Acesso em: 04 março de 2021.

OLIVEIRA, M. P.; SILVA, I. C. M.; ALBUQUERQUE, G. G. Pesquisa científica no curso de Enfermagem: revisão integrativa. Revista Práxis. v.8, no 16. pág. 115-121. Dezembro, 2016. Disponível em: http://revistas.unifoa.edu.br/index.php/praxis/article/viewFile/804/634. Acesso em: 13 de março de 2021. 


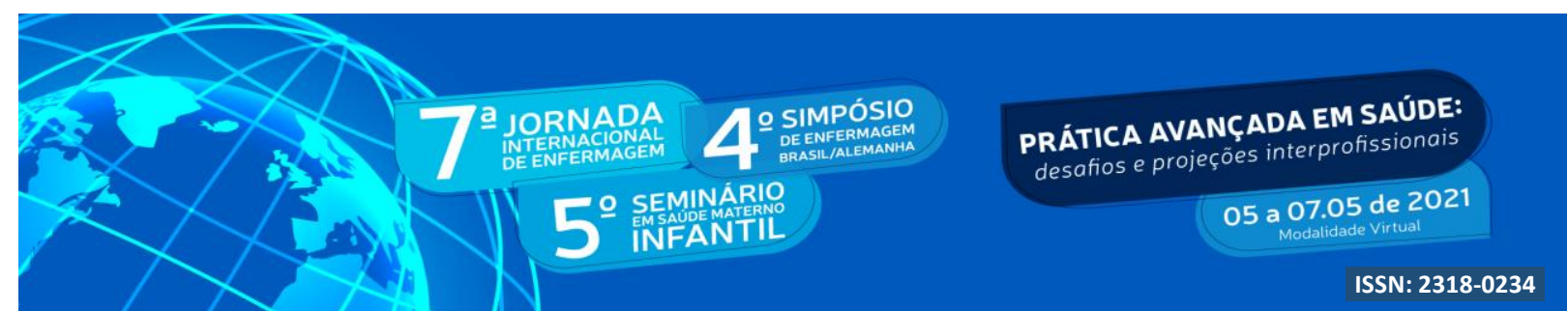

PEREIRA, M. J.; SILVA, A. P.; OLIVEIRA, M. L.; LIMA, T.; SILVA, P. O.; NUNES, L. O. A Importância da Iniciação Científica para alunos de Graduação em Biomedicina. Jornada Científica da Faculdade São Lourenço. 2015. Disponível em:

https://portal.unisepe.com.br/unifia/wpcontent/uploads/sites/10001/2018/06/023 inciacao cientifica aluno biomedicina.pdf. Acesso em: 03 março de 2021.

PINHO, M. J. Ciência e ensino: contribuições da iniciação científica na educação superior. Avaliação: Revista da Avaliação da Educação Superior (Campinas). v. 22, nº 3, pág. 658675, novembro, 2017. Disponível em: https://www.scielo.br/scielo.php?pid=S141440772017000300658\&script=sci_arttext. Acesso em: 08 março de 2021.

PINTO, N. L. S.; FERNANDES, L. M. A.; SILVA, F. F. Para além da formação acadêmica: as contribuições da iniciação científica para o desenvolvimento pessoal e profissional de estudantes da área de administração. Administração: Ensino e Pesquisa. v. 17, no 2, pág. 301-325, Janeiro, 2016. Disponível em:

https://media.proquest.com/media/hms/ORIG/1/vburB? s=e6RLBffhsU2093RjSoGwb0ZLC2 E\%3D. Acesso em: 09 de março 2021.

QUEVEDO, C; GIMENEZ, F. V. M. Importância da Iniciação Científica na formação de enfermeiros. Revista Científica Eletrônica de Enfermagem da FAEF. v. 2, no 1, Fevereiro, 2019. Disponível em: http://faef.revista.inf.br/imagens_arquivos/arquivos_destaque/5i6VBQ7Fui3B7Js_2019-1112-21-36-49.pdf. Acesso em: 03 março de 2021.

SANTOS, V. C.; ANJOS, K. F.; ALMEIDA, O. S. Iniciação Científica a partir de estudantes de Enfermagem. Revista Brasileira de Ciências da Saúde. v. 19, nº 4, pág. 255-260, 2015. Disponível em:

https://pdfs.semanticscholar.org/889b/5614fb2fcfc33a10d7a6c8af84cca4a592da.pdf. Acesso em: 08 março de 2021.

SOBRAL, H. C. F.; SANTOS, I. H. F.; TORALES, A. P. B. Relato De Experiência: A Iniciação Científica na Vida Acadêmica. Encontro Internacional de Formação de Professores e Fórum Permanente de Inovação Educacional. v. 9, nº 1, pág. 1-12, 2016. Disponível em: https://eventos.set.edu.br/enfope/article/view/2450. Acesso em: 08 de março 2021. 